\title{
ВАРИАТИВНОСТЬ ТЕРМИНОЛОГИЧЕСКОЙ ДЕФИНИЦИИ В АНГЛОЯЗЫЧНОМ ЛИНГВИСТИЧЕСКОМ И ИННОВАЦИОННО-ТЕХНИЧЕСКОМ ДИСКУРСЕ
}

\author{
Т. В. Аксенова
}

Мордовский государственный университет имени Н. П. Огарёва

\section{VARIABILITY OF TERMINOLOGICAL DEFINITION IN ENGLISH LINGUISTIC AND INNOVATIVE TECHNICAL DISCOURSE}

\author{
T. V. Aksenova \\ Ogarev Mordovia State University
}

\begin{abstract}
Аннотация: большое количество современных гуманитарных исследований посвящено проблеме дискурса и рассмотрению языковых явлений в их речевом проявлении. В частности, интерес для изучения представляет собой научный дискурс как сфера терминопорождения. На сегодняшний день вопрос терминологической и дефиниционной вариативности является актуальным в ряде исследований, однако до настоящего момента не проводился сравнительно-сопоставительный анализ специфики и причин вариативности в разных типах дискурса. Целью данного исследования является рассмотрение англоязычного лингвистического и инновачионно-технического типов дискурса как особой среды терминообразования и выявление характерных особенностей вариативности терминологической дефиниции в обоих типах дискурса. В статье проводится анализ словарных и контекстуальных дефиниций наиболее употребительных терминов англоязычного лингвистического и инновачионно-технического типов дискурса. В иелях достижения наиболее эффективных результатов в качестве материалов исследования были выбраны описательный и сопоставительно-стилистический метод, а также метод контекстуального и лингвостилистического анализа. Были рассмотрены дефиниции терминов в универсальных и специализированных толковых словарях, а также в текстах научных работ. Отмечено, что дефиниционная вариативность наблюдается в обоих типах дискурса. Были выявлены различия между словарными и контекстуальными терминологическими дефинициями, а также между словарными статьями в универсальных и специализированных словарях. Кроме того, описываются специфические особенности вариативности в анализируемых типах дискурса. В заключение делаются выводы о причинах возникновения вариативности в англоязычном лингвистическом и инновационно-техническом типах дискурса. Отмечаются перспективы исследования в данном направлении.
\end{abstract}

Ключевые слова: дефиниция, вариативность, лингвистический дискурс, инноваџионно-технический дискурс, нанотехнологии.

\begin{abstract}
: a large number of modern humanitarian studies are devoted to the problem of discourse and the consideration of linguistic phenomena in their speech manifestation. In particular, the scientific discourse as a sphere of term formation is of interest for study. To date, the issue of terminological and definitional variability has been relevant in a number of studies, but until now a comparative analysis of the specificity and causes of variability in different types of discourse has not been carried out. The purpose of this study is to consider the English-language linguistic and innovative-technical types of discourse as a special environment for term formation and to identify the characteristic features of definitional variability in both types of discourse. The article analyzes the dictionary and contextual definitions of the most common terms in the English-language linguistic and innovative-technical types of discourse. In order to achieve the most effective results, the descriptive and comparative-stylistic method, as well as the method of contextual and stylistic analysis, were chosen. Definitions
\end{abstract}

(C) Аксенова Т. В., 2020

Контент доступен под лицензией Creative Commons Attribution 4.0 License.

The content is available under Creative Commons Attribution 4.0 License. 
of terms were considered in universal and specialized explanatory dictionaries, as well as in the texts of scientific papers. Definitional variability is observed in both types of discourse. Differences are identified between vocabulary and contextual terminological definitions, as well as between vocabulary entries in universal and specialized dictionaries. In addition, the specific features of variability in the analyzed types of discourse are described. Conclusions are drawn about the causes of variability in the English-language linguistic and innovative-technical types of discourse. The author also outlines the perspectives of research.

Key words: definition, variability, linguistic discourse, innovative technical discourse, nanotechnology.

В настоящее время значительное количество научных работ посвящено понятиям речи и дискурса в связи с тем, что исследователи все чаще обращаются к парадигмальному рассмотрению языковых явлений. На сегодняшний день существует большое количество критериев классификации дискурса, в частности, по принадлежности к определенному профессиональному сообществу [1]. В рамках данной типологии выделяется научный дискурс, который послужил материалом данного исследования, поскольку именно в научном дискурсе наблюдаются процессы терминообразования.

Научный дискурс характеризуется информативностью, логичностью и точностью изложения [2, c. 128], в том числе и в терминологических дефинициях, являющихся одним из ключевых компонентов научного текста. Однако зачастую в словарях и научных работах наблюдается дефиниционная вариативность, рассмотрению которой был посвящен ряд научных работ $[3 ; 4]$.

Изучая вариативность терминологической дефиниции в англоязычном инновационно-техническом дискурсе [5], мы задались вопросом, является ли подобное явление характерным для иных дисциплинарных областей. В качестве примера был выбран лингвистический дискурс, поскольку он относится к принципиально иной области знания - гуманитарной. Рассматриваемые типы дискурса отличаются друг от друга по ряду параметров. Во-первых, это предмет исследования. Инновационно-технический дискурс фиксирует результаты познания объектов реальной действительности, в то время как в центре внимания лингвистического дискурса находится язык как система знаков, вербализуемая в устной или письменной форме. Во-вторых, вышеуказанные типы дискурса отличаются продолжительностью существования и, как следствие, степенью изученности дисциплинарной области. Ввиду этого полагаем, что за терминологической вариативностью в обоих случаях кроются разные причины, которые будут освещены в рамках данной работы.

Анализу подверглись как словарные, так и контекстуальные дефиниции наиболее употребительных терминов англоязычного сегмента лингвистического и инновационно-технического дискурса. Исследование проводилось путем описательного и сопоставительно-стилистического метода, а также метода контекстуального и лингвостилистического анализа.

В качестве примера терминологической вариативности в англоязычном лингвистическом дискурсе был выбран термин discourse, поскольку, несмотря на длительность его активного функционирования в научном диалоге, ученые так и не сошлись в едином мнении относительно его дефиниционных характеристик.

Термин дискурс имеет множество лингвистических определений, в основе которых лежит стремление ученых уточнить традиционные представления о речи, тексте и диалоге. История представленной точки зрения связана с именем американского ученого 3. Харриса, который в 1952 г. ввел терминологическое сочетание «дискурс-анализ». Под ним исследователь подразумевал формальный метод, заключающийся в выявлении частотности употребления значимых единиц в тексте [6, p. 1]. При этом под дискурсом 3. Харрис понимал «последовательность предложений, произнесенную (или написанную) одним (или более) человеком в определенной ситуации» («the sentences spoken or written in succession by one or more persons in a single situation») [6, p. 3]. Со времени первого появления термина «дискурс» в лингвистическом дисциплинарном контексте он приобрел большое количество оттенков значения. В том числе в современной лингвистике дискурс часто понимается как «сложное коммуникативное явление, включающее, кроме текста, еще и экстралингвистические факторы (знания о мире, мнения, установки, цели адресата), необходимые для понимания текста» $[7$, с. 8], как речь, «погруженная в жизнь» $[8$, с. 136$137]$.

В результате анализа англоязычных словарных дефиниций термина discourse необходимо отметить, что в целом они варьируются в зависимости от того, является ли толковый словарь универсальным (предназначенным для широкого круга читателей) или специализированным (для представителей определенной сферы знания). Так, в универсальных словарях приводится несколько (от 2 до 5) дефиниций, из которых в ряде изданий одна имеет пометку Linguistics. В качестве доказательства вариативности словарной дефиниции приведем примеры лингвистических определений термина discourse из универсальных словарей: 
Linguistics. any unit of connected speech or writing longer than a sentence [9];

a linguistic unit (such as a conversation or a story) larger than a sentence [10];

Linguistics. A connected series of utterances; a text or conversation [11].

Данные дефиниции имеют схожие компоненты (unit, connected, longer/larger than a sentence, conversation), однако в целом, транслируя одно значение, имеют разную лексическую оболочку. Во всех приведенных выше примерах акцент делается на устную или письменную форму речи, а также на протяженность отрезка, рассматриваемого как дискурс. В других словарных определениях из англоязычных универсальных словарей можно встретить несколько иную трактовку понятия discourse:

In linguistics, discourse is natural spoken or written language in context, especially when complete texts are being considered [12];

linguistics written or spoken language, especially when it is studied in order to understand how people use language [13].

Вышеуказанные дефиниции дополняются экстралингвистическим компонентом, т. е. в определение включается понятие контекста и упоминаются обстоятельства, в которых используется тот или иной речевой фрагмент. Следует отметить, что в универсальных словарях, широко охватывающих значения термина, а следовательно, в большинстве случаев не указывающих отдельные нюансы его семантики, таких примеров значительно меньше (около 20 \%).

Говоря о специализированных словарях, следует отметить их особенность актуализации терминологической дефиниции: она значительно обширнее дефиниции, представленной в универсальном словаpe, за счет включения полных предложений, призванных подробнее характеризовать определяемую лексическую единицу:

discourse, generic term for various types of text. The term has been used with various differences in meaning... [14];

discourse, any coherent succession of sentences, spoken or (in most usage) written. Thus this entry in the dictionary is an example of discourse [15].

Помимо этого, часто термин discourse определяется через его противопоставление термину text, так, к примеру:

Discourse is linguistic communication seen as a transaction between speaker and hearer, as an interpersonal activity whose form is determined by its social purpose. Text is linguistic communication (either spoken or written) seen simply as a message coded in its auditory or visual medium [16].

Discourse analysis focusses on the structure of naturally occurring spoken language, as found in such «discourses» as conversations, interviews, commentaries, and speeches. Text analysis focusses on the structure of written language, as found in such «texts» as essays, notices, road signs, and chapters [17].

Посредством противопоставления двух понятий, которые сближаются в ряде теорий, дефиниция термина в специализированных словарях становится еще более протяженной, но в то же время охватывает больше дефиниционных нюансов.

Вследствие этого можно говорить о том, что специализированные лингвистические словари отличаются большей вариативностью по сравнению с универсальными толковыми словарями, во-первых, за счет развернутого характера словарных статей, а во-вторых, из-за различия в авторской точке зрения на описываемый феномен. В этом проявляется их сходство с контекстуальными дефинициями, представленными в статьях, которые, в свою очередь, еще более многообразны и имеют свою специфику.

В ряде случаев авторы прибегают к цитированию словарных дефиниций, например, С. Миллз в книге с одноименным названием «Discourse» приводит дефиниции базового термина из Collins Concise English Dictionary, Longman Dictionary of the English Language и Collins Robert Concise French Dictionary c целью продемонстрировать эволюцию данного понятия:

...if we take even the simplest route through its [the general use of the term] history we can see a shifting from the highlighting of one aspect of usage to another [18].

Еще в одном примере цитирования словарной дефиниции автор ссылается на Oxford Concise Dictionary of Linguistics, но при этом слова «in other words» - «другими словами»- подчеркивают тот факт, что словарная дефиниция используется лишь в качестве подтверждения позиции автора:

In other words discourse is 'any coherent succession of sentences, spoken or written' (Matthews, 2005:100) [19].

Собственно авторская дефиниция, приведенная в начале работы, значительно более развернутая:

Discourse is the creation and organization of the segments of a language above as well as below the sentence. $[. .$.$] the adduced meaning is always beyond the$ sentence. The term discourse applies to both spoken and written language... [...] Discourse cannot be confined to sentential boundaries. It is something that goes beyond the limits of sentence [19].

Данная контекстуальная дефиниция значительно объемнее определений, представленных в словарях, поскольку сложность и неоднозначность понятия discourse практически невозможно передать в коротком текстовом отрезке. Формат научной статьи позволяет автору уделить внимание большому количеству значений рассматриваемого термина, а также подчер- 
кнуть, во-первых, организацию дискурса, во-вторых, его протяженность, в-третьих, письменную или устную форму речи и, наконец, в-четвертых, смысл, который выходит далеко за рамки предложения.

Иногда авторы в локальном контексте своей работы приводят определения дискурса как лексемы естественного языка и как лингвистического термина, тем самым наглядно показывая разницу между ними:

«discourse» with a little «d», to me, means connected stretches of language that make sense, so «discourse» is a part of «Discourse» [20].

Таким образом, при рассмотрении дефиниций термина discourse из различных источников (универсальных толковых словарей, специализированных лингвистических словарей и научных статей) можно прийти к выводу, что им свойственна вариативность, причем чаще всего она наблюдается в специализированных словарях и тексте научных статей. Причиной тому, в первую очередь, служит различие авторских точек зрения на феномен дискурса. В ходе развития научной мысли понятие дискурса эволюционировало, приобретая новые оттенки значения, однако при этом в лингвистической практике закрепилось и первоначальное понимание дискурса, что привело к множественности его значений и вариативности терминологической дефиниции.

В рамках англоязычного инновационно-технического дискурса для дефиниционного анализа был выбран термин nanotechnology, который, наряду с термином discourse в лингвистике, является одним из наиболее употребительных в своей области знания. История существования термина nanotechnology несколько короче, чем термина discourse. Впервые он был введен в употребление японским исследователем Н. Танигучи в 1974 г. и означал манипуляции с материалами, размер которых менее одного микрона («the processes of separation, consolidation, and deformation of materials by one atom or one molecule» [21]). С того времени определение нанотехнологий в основном осталось неизменным. Что примечательно, и в универсальных, и в специализированных словарях одним из ключевых аспектов дефиниции выступает размер объектов и манипуляции с ними на атомном или молекулярном уровнях:

the manipulation of materials on an atomic or molecular scale especially to build microscopic devices (such as robots) [10];

the branch of technology that deals with dimensions and tolerances of less than 100 nanometres, especially the manipulation of individual atoms and molecules [11];

the engineering of functional systems at the molecular scale [22];

the understanding and control of matter at the nanoscale, at dimensions between approximately 1 and 100 nanometers, where unique phenomena enable novel applications [23].

Вышеприведенные примеры, взятые из универсальных и специализированных словарей, доказывают тот факт, что термин nanotechnology не имеет ключевых дефиниционных отличий. Вариативность затрагивает упоминание размеров объектов: в ряде случаев данная информация опускается, однако всегда фигурирует атомный или молекулярный уровень. Наблюдаются отличия в родовом понятии дефиниенса (определяющей части) (manipulation, engineering, understanding and control). Кроме того, в ряде дефиниций фигурирует дополнительная информация, а именно цель нанотехнологий (to build microscopic devices) или специфика материалов на наноуровне (where unique phenomena enable novel applications). Однако следует подчеркнуть, что, в отличие от термина discourse, дефиниционная вариативность термина nanotechnology в словарях в основном относится к плану выражения, нежели к плану содержания, что свидетельствует о единодушии исследователей в вопросах понятия нанотехнологий.

Переходя к контекстуальным дефинициям термина nanotechnology, стоит отметить еще одно существенное отличие лингвистического от инновационно-технического дискурса: это распределение текстов согласно собственно научному и научно-популярному подстилям. Область наноисследований является одним из передовых научных направлений на сегодняшний день, что делает ее интересной для широкой публики. Этим, на наш взгляд, объясняется большое количество научно-популярной литературы на эту тему. В то же время объектом изучения лингвистики являются язык и речь, существующие многие сотни лет, и лингвистические исследования в основном направлены на развитие методологии анализа уже существующего феномена, а не на изобретение и создание новых объектов. Очевидно, что меньшее количество людей заинтересовано в подобных исследованиях нематериального проявления механизма мышления, что находит отражение в значительно меньшем количестве научно-популярных лингвистических текстов. Таким образом, вариативность контекстуальной дефиниции термина nanotechnology преимущественно рассматривалась в сравнении собственно научного и научно-популярного подстилей.

В первую очередь, необходимо сказать, что в текстах собственно научного подстиля термин папоtechnology крайне редко сопровождается дефиницией (7 \% случаев), поскольку принадлежит к числу базовых и не нуждается в пояснении экспертам данной области. Тем не менее можно встретить подобные примеры: 
Nanotechnology is a rapidly advancing and truly multidisciplinary field involving subjects such as physics, chemistry, engineering, electronics and biology. Its aim is to advance science at atomic and molecular level in order to make materials and devices with novel and enhanced properties. The potential applications are exceptionally diverse and beneficial, ranging from self-cleaning windows and clothes to ropes to tether satellites to the earth's surface, to new medicines [24].

Данная дефиниция указывает на базовые характеристики термина, отмечаемые и в словарях: уровень манипуляции с объектами (atomic and molecular level) и цель нанотехнологий (to make materials and devices with novel and enhanced properties). Вместе с тем автор расширяет определение, упоминая смежные научные дисциплины (physics, chemistry, engineering, electronics and biology) и потенциальную область применения достижений науки (from self-cleaning windows and clothes to ropes to tether satellites to the earth's surface, to new medicines). В этом случае определения термина nanotechnology структурно сходны с определениями термина discourse: они указывают основные характеристики понятия и более подробно описывают отдельные его нюансы.

Что касается научно-популярного подстиля, то в нем базовые термины инновационно-технического дискурса, в том числе nanotechnology, практически всегда сопровождаются дефиницией (95 \% случаев):

Nanotechnology is a field of research and innovation concerned with building 'things' ... on the scale of atoms and molecules. A nanometre is one-billionth of a metre: ten times the diameter of a hydrogen atom. The diameter of a human hair is, on average, 80,000 nanometres [25];

Nanotechnology is the study and manipulation of matter at incredibly small sizes, generally between one and 100 nanometers. To put this in perspective, a piece of paper is about 100,000 nanometers thick [26].

В целом, дефиниции термина nanotechnology в научно-популярном стиле по затрагиваемым характеристикам практически не отличаются от словарных или собственно научных дефиниций. Основное отличие заключается в пояснении размеров наноуровня и сравнении их с такими осязаемыми объектами, как человеческий волос или бумага. Для экспертов в области нанотехнологий подобное уточнение было бы излишне, как, впрочем, и определение нанотехнологий в целом.

В ряде случаев авторы научно-популярного текста используют неформальный стиль речи, чтобы сделать текст более привлекательным для читателя, не имеющего отношения к инновационным технологиям:

Nanotechnology is the application of these nanostructures into useful nanoscale devices. ... To explain that, it's important to understand that the nanoscale isn't just small, it's a special kind of small [27].
Nanotechnology is the study of what happens when things get very, very small - only a few atoms in size [28].

Так, в обоих примерах авторы подчеркивают размер наноуровня с помощью элементов разговорного стиля (isn't just small, it's a special kind of small; when things get very, very small).

Подводя итог анализу дефиниций термина nanotechnology в словарях и тексте научных работ, можно сказать, что их вариативность преимущественно затрагивает форму, посредством которой актуализуется определение. Авторы могут дополнять дефиницию информацией, которая при этом не меняет ее сути, либо прибегать к неформальному стилю речи с целью привлечения более широкой аудитории.

На основании всего вышесказанного можно сделать следующие выводы относительно вариативности терминологической дефиниции в англоязычном лингвистическом и инновационно-техническом дискурсе. Во-первых, выбор ключевых терминов обеих областей знания позволил проанализировать большое количество примеров их определений как в словарях (универсальных и специализированных), так и в тексте научных работ. Во-вторых, независимо от продолжительности функционирования термина его дефиниция вариативна. В-третьих, дефиниционная вариативность терминов discourse и nanotechnology различна. В лингвистическом дискурсе речь в основном идет о парадигмальных отличиях, т. е. дефиниция отражает принципиальные отличия в авторских точках зрения на характеризуемый феномен. В случае с инновационно-техническим дискурсом наблюдаются изменения в плане выражения соответственно целевой аудитории. Большое количество текстов области нанотехнологий написано в научно-популярном стиле, что приводит к значительным различиям в плане выражения, однако это практически не затрагивает план содержания.

Говоря о перспективах исследования, следует отметить, что сложно прогнозировать поведение терминологической дефиниции в обоих типах дискурса применительно к менее распространенным или вновь появляющимся терминам. Вследствие этого представляет интерес их сравнительно-сопоставительный анализ, а также рассмотрение терминологической вариативности в дискурсах, смежных с лингвистической и инновационно-технической областями знания.

\section{ЛИТЕРАТУРА}

1. Данилова С. А. Типология дискурса // Гуманитарные, социально-экономические и общественные науки. 2015. № 1. С. 345-349.

2. Зорина Ю. В. К вопросу о некоторых специфических чертах научного дискурса // Международный на- 
учно-исследовательский журнал. 2018. № 8 (74). C. $128-131$.

3. Григорьев Г. В., Ручкина Е. М., Васильев Л. Г. Состояние развития понятийно-структурной организации терминологии через призму парадигматических отношений и вариативности терминологических дефиниций // Филологические науки. Вопросы теории и практики. 2019. Т. 12. № 4. С. 244-251.

4. Кантышева Н. Г. Дефиниционная и контекстуальная многозначность термина «номенклатура» // Вестник Тюмен. гос. ун-та. Гуманитарные исследования. Humanitates. 2015. T. 1. № 4 (4). C. 83-92.

5. Свойкин К. Б., Яшина Т. В. Инновационно-техническая терминология в аспекте субтекста адресации // Гуманитарные науки и образование. 2014. № 3 (19). C. 141-145.

6. Harris Z. Discourse analysis // Language. 1952. V. 28, № 1. Pp. 1-30.

7. Караулов Ю. Н., Петров В. В. От грамматики текста к когнитивной теории дискурса // Язык. Познание. Коммуникация. М. : Прогресс, 1989. С. 5-11.

8. Арутюнова Н. Д. Дискурс // Лингвистический энциклопедический словарь. М. : Сов. Энциклопедия, 1990. C. 136-137.

9. Dictionary.com. URL: https://www.dictionary.com

10. Merriam-Webster. URL: https://www.merriam-webster.com

11. English Oxford Living Dictionaries. URL: https:// en.oxforddictionaries.com

12. Collins. Pioneers in dictionary publishing since 1819. URL: https://www.collinsdictionary.com

13. Macmillan Dictionary. URL: https://www.macmillandictionary.com

14. Bussmann H. Routledge Dictionary of Language and Linguistics. Taylor \& Francis e-Library, 2006. 1335 p. URL: https://www.e-reading.club/bookreader.php/142124/ Routledge_Dictionary_of_Language_and_Linguistics.pdf

15. Matthews P. H. Oxford Concise Dictionary of Linguistics. Oxford University Press, 2007. 443 p.

16. Hawthorn J. A Concise Glossary of Contemporary Literary Theory. Arnold, London, 1992. 288 p.

17. Crystal D. The Cambridge Encyclopaedia of Language. Cambridge University Press, 1987. 472 p.

18. Mills $S$. Discourse. Taylor \& Francis e-Library, 2001. 188 p. URL: https://analepsis.files.wordpress.com/2011/08/ discourse-sara-mills.pdf

19. Sharma V. K., Sharma M. K. Linguistic discourse analysis: Introduction and structure // Call for papers. 2010. URL: http://call-for-papers.sas.upenn.edu/node/39688

20. Gee J. P. Literacy, discourse and linguistics // Journal of Education. 1989. Vol. 171, No. 1. Pp. 5-14.

21. Taniguchi N. On the Basic Concept of «Nano-Technology» // Proc. Intl. Conf. Prod. Eng. Tokyo. Part II. Japan Society of Precision Engineering, 1974. URL: http://library. thinkquest.org/05aug/01179/norio.html

22. What is nanotechnology? // Center for Responsible Nanotechnology. URL: http://www.crnano.org/whatis.htm
23. National Nanotechnology Initiative. URL: http:// www.nano.gov/

24. From Nautilus to nanobo(a)ts: the visual construction of nanoscience // AzoNano. URL: https://www.azonano.com/article.aspx?ArticleID $=1466$

25. What is nanotechnology and what can it do? // AzoNano. URL: https://www.azonano.com/article.aspx?ArticleID $=1134$

26. ChemicalSafetyFacts.org. URL: https://www.chemicalsafetyfacts.org/nanotechnology/

27. Ratner M. Ratner D. Nanotechnology: A gentle introduction to the next big idea. Prentice Hall, New Jersey, 2002. $133 \mathrm{p}$.

28. What is nanotechnology? // Science Friday. URL: https://www.sciencefriday.com/educational-resources/ what-is-nanotechnology/

\section{REFERENCES}

1. Danilova S. A. Tipologiya diskursa [Typology of discourse]. In Gumanitarnye, sotsial'no-ekonomicheskie $i$ obshchestvennye nauki [Humanities, Social-Economic and Social Sciences]. 2015. № 1. Pp. 345-349.

2. Zorina Yu. V. K voprosu o nekotorykh spetsificheskikh chertakh nauchnogo diskursa [To the question of some specific features of scientific discourse]. In Mezhdunarodnyj nauchno-issledovatel'skij zhurnal [International Research Journal]. 2018. № 8 (74). Pp. 128-131.

3. Grigor'ev G. V., Ruchkina E. M., Vasil'ev L. G. Sostoyanie razvitiya ponyatiyno-strukturnoj organizatsii terminologii cherez prizmu paradigmaticheskikh otnoshenij i variativnosti terminologicheskikh definitsij [The state of development of the conceptual and structural organization of terminology through the prism of paradigmatic relations and variability of terminological definitions]. In Filologicheskie nauki. Voprosy teorii i praktiki [Philological Sciences. Issues of Theory and Practice]. 2019. Vol. 12, No. 4. Pp. 244-251.

4. Kantysheva N. G. Definitsionnaya i kontekstual'naya mnogoznachnost' termina «nomenklatura» [Definitional and contextual polysemy of the term "nomenclature"]. In Vestnik Tyumenskogo gosudarstvennogo universiteta. Gumanitarnye issledovaniya. Humanitates [Tyumen State University Herald. Modern Humanitarian Research. Humanitates]. 2015. Vol. 1. № 4 (4). Pp. 83-92.

5. Svojkin K. B., Yashina T. V. Innovatsionno-tekhnicheskaya terminologiya $\mathrm{v}$ aspekte subteksta adresatsii [Innovative technical terminology in the aspect of the subtext of addressing]. In Gumanitarnye nauki i obrazovanie [The Humanities and Education]. 2014. № 3 (19). Pp. 141-145.

6. Harris Z. Discourse analysis. In Language. 1952. Vol. 28. № 1. Pp. 1-30.

7. Karaulov Yu. N., Petrov V. V. Ot grammatiki teksta k kognitivnoj teorii diskursa [From text grammar to cognitive theory of discourse]. In Yazyk. Poznanie. Kommunikatsiya [Language. Cognition. Communication]. Moscow, Progress, 1989. Pp. 5-11. (In Russ.). 
8. Arutyunova N. D. Diskurs [Discourse] In Lingvisticheskij ehntsiklopedicheskij slovar' [Linguistic Encyclopedic Dictionary]. Moscow, Sov. Ehntsiklopediya, 1990. Pp. 136-137.

9. Dictionary.com. Available at: https://www.dictionary. com

10. Merriam-Webster. Available at: https:/www.merriam-webster.com

11. English Oxford Living Dictionaries. Available at: https://en.oxforddictionaries.com

12. Collins. Pioneers in dictionary publishing since 1819. Available at: https://www.collinsdictionary.com

13. Macmillan Dictionary. Available at: https://www. macmillandictionary.com

14. Bussmann H. Routledge Dictionary of Language and Linguistics. Taylor \& Francis e-Library, 2006, 1335 p. Available at: https://www.e-reading.club/bookreader. php/142124/Routledge_Dictionary_of_Language_and_Linguistics.pdf

15. Matthews P. H. Oxford Concise Dictionary of Linguistics. Oxford University Press, 2007, 443 p.

16. Hawthorn J. A Concise Glossary of Contemporary Literary Theory. Arnold, London, 1992, 288 p.

17. Crystal D. The Cambridge Encyclopaedia of Language. Cambridge University Press, 1987, 472 p.

18. Mills S. Discourse. Taylor \& Francis e-Library, 2001, 188 p. Available at: https://analepsis.files.wordpress. com/2011/08/discourse-sara-mills.pdf

Мордовский государственньй университет имени Н. П. Огарёва

Аксенова Т. В., кандидат филологических наук, доиент кафедры английской филологии

E-mail: tatiana.aksenova88@mail.ru

Поступила в редакциию 31 августа 2019 г.

Принята к публикаџии 27 декабря 2019 г.

\section{Для цитирования:}

Аксенова T. В. Вариативность терминологической дефиниции в англоязычном лингвистическом и инновационно-техническом дискурсе // Вестник Воронежского государственного университета. Серия: Лингвистика и межкультурная коммуникация. 2020. № 1. С. 70-76. DOI: https://doi.org/10.17308/lic.2020.1/2732
19. Sharma V. K. Linguistic discourse analysis: Introduction and structure. In Call for papers, 2010. Available at: http://call-for-papers.sas.upenn.edu/node/39688

20. Gee J. P. Literacy, discourse and linguistics. In Journal of Education.1989. Vol. 171. No 1. Pp. 5-14.

21. Taniguchi N. On the Basic Concept of «Nano-Technology». In Proc. Intl. Conf. Prod. Eng. Tokyo. Part II. Japan Society of Precision Engineering, 1974. Available at: http:// library.thinkquest.org/05aug/01179/norio.html

22. What is nanotechnology? In Center for Responsible Nanotechnology. Available at: http://www.crnano.org/whatis.htm

23. National Nanotechnology Initiative. Available at: http://www.nano.gov/

24. From Nautilus to nanobo(a)ts: the visual construction of nanoscience. In AzoNano. Available at: https://www. azonano.com/article.aspx?ArticleID $=1466$

25. What is nanotechnology and what can it do? In AzoNano. Available at: https://www.azonano.com/article. aspx?ArticleID $=1134$

26. ChemicalSafetyFacts.org. Available at: https://www. chemicalsafetyfacts.org/nanotechnology/

27. Ratner M. Nanotechnology: A gentle introduction to the next big idea. Prentice Hall, New Jersey, 2002. 133 p.

28. What is nanotechnology? In Science Friday. Available at: https://www.sciencefriday.com/educational-resources/what-is-nanotechnology/

Ogarev Mordovia State University

Aksenova T. V., Candidate of Philology, Associate Professor of the English Philology

E-mail: tatiana.aksenova88@mail.ru

Received: 31 August 2019

Accepted: 27 December 2019

\section{For citation:}

Aksenova T. $V$. Variability of terminological definition in english linguistic and innovative technical discourse. Proceedings of Voronezh State University. Series: Linguistics and Intercultural Communication. 2020. No. 1. Pp. 70-76. DOI: https://doi.org/10.17308/lic.2020.1/2732 